\title{
On the link pattern distribution of quarter-turn symmetric FPL configurations
}

\author{
Philippe Duchon ${ }^{\dagger}$ \\ Philippe Duchon - ENSEIRB - LaBRI, Université Bordeaux 1, 351 cours de la Libération, F-33405 Talence
}

\begin{abstract}
We present new conjectures on the distribution of link patterns for fully-packed loop (FPL) configurations that are invariant, or almost invariant, under a quarter turn rotation, extending previous conjectures of Razumov and Stroganov and of de Gier. We prove a special case, showing that the link pattern that is conjectured to be the rarest does have the prescribed probability. As a byproduct, we get a formula for the enumeration of a new class of quasi-symmetry of plane partitions.

Résumé. Nous présentons de nouvelles conjectures portant sur la distribution des schémas de couplage des configurations de boucles compactes (FPL) invariantes, ou presque invariantes, par une rotation d'un quart de tour. Ces nouvelles conjectures étendent des conjectures précédentes dues à Razumov et Stroganov et à de Gier. Dans chaque cas, nous prouvons un cas particulier, en démontrant que le schéma de couplage conjecturé pour être le plus rare a effectivement la probabilité prédite. Nous obtenons également une formule pour l'énumération d'une nouvelle classe de quasi-symétrie de partitions planes.
\end{abstract}

Keywords: fully packed loop model, rhombus tilings, plane partitions, nonintersecting lattice paths

\section{Introduction}

In this paper, we study configurations in the fully packed loop model, or, equivalently, alternating-sign matrices, that are invariant or almost invariant under a rotation of 90 degrees. While the enumeration of this symmetry class of alternating-sign matrices was conjectured by Robbins [23] and proved by $\mathrm{Ku}$ perberg [15] and Razumov and Stroganov [21], their refined enumeration according to the link patterns of the corresponding fully packed loop configurations seems to have avoided notice so far. We conjecture very close connections between this refined enumeration and the corresponding enumeration for half-turn invariant configurations, as studied by de Gier [7]. This is yet another example of a "RazumovStroganov-like" conjecture, suggesting a stronger combinatorial connection between fully-packed loop configurations and their link patterns than originally conjectured in [20].

The paper is organized as follows. In Section 2, we recall a number of definitions and conjectures on FPLs and their link patterns, and define a new class of "quasi-quarter-turn-invarriant" FPLs when the size

\footnotetext{
${ }^{\dagger}$ Research supported by French ANR project MARS (BLAN06-2-134516). 2000 Mathematical Subject Classification: Primary 05A15; Secondary 05B45, 82B20
} 
is an even integer of the form $4 n+2$. We formulate a conjecture on the enumeration of these qQTFPLs. In Section 3, we give new conjectures on the distribution of link patterns of QTFPLs and qQTFPLs; these can be seen as natural extensions of the previously known Razumov-Stroganov and de Gier conjectures on general and half-turn symmetric FPLs, respectively. We prove special cases of our conjectures in Section 4; in the qQTFPL case this is achieved by making an explicit connection with the enumeration of some new class of plane partitions.

\section{Fully-packed loops and link patterns}

\subsection{Fully-packed loop configurations}

A fully-packed loop configuration (FPL for short) of size $N$ is a subgraph of the $N \times N$ square lattice ${ }^{(i)}$, where each internal vertex has degree exactly 2, forming a set of closed loops and paths ending at the boundary vertices. The boundary conditions are the alternating conditions: boundary vertices also have degree 2 when boundary edges (edges that connect the finite square lattice to the rest of the $\mathbb{Z}^{2}$ lattice) are taken into account, and these boundary edges, when going around the grid, are alternatingly "in" and "out" of the FPL. For definiteness, we use the convention that the top edge along the left border is always "in". Thus, exactly $2 N$ boundary edges act as endpoints for paths, and the FPL consists of $N$ noncrossing paths and an indeterminate number of closed loops.

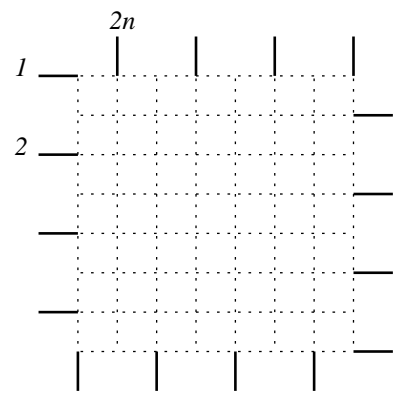

(a)

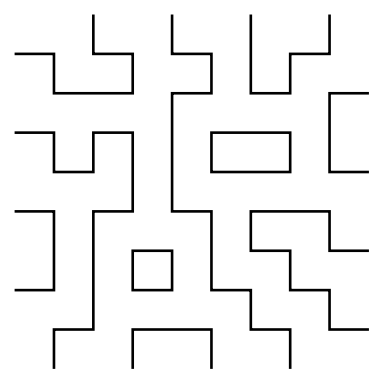

(b) $\begin{array}{llllllll}0 & 0 & 0 & 0 & 0 & 1 & 0 & 0\end{array}$

$\begin{array}{llllllll}0 & 1 & 0 & 0 & 0 & 0 & 0 & 0\end{array}$

$\begin{array}{llllllll}0 & 0 & 0 & 1 & 0 & -1 & 0 & 1\end{array}$

$\begin{array}{llllllll}0 & 0 & 1 & -1 & 0 & 1 & 0 & 0\end{array}$

$\begin{array}{llllllll}0 & 0 & 0 & 0 & 0 & 0 & 1 & 0\end{array}$

$\begin{array}{llllllll}1 & -1 & 0 & 0 & 1 & 0 & 0 & 0\end{array}$

$\begin{array}{llllllll}0 & 1 & 0 & 0 & 0 & 0 & 0 & 0\end{array}$

$\begin{array}{llllllll}0 & 0 & 0 & 1 & 0 & 0 & 0 & 0\end{array}$

(c)

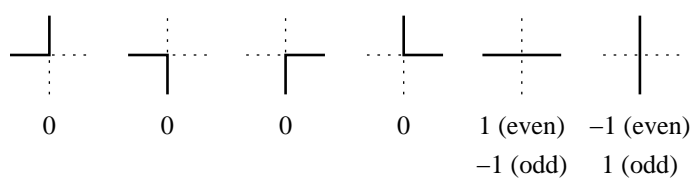

(d)

Fig. 1: (a) boundary conditions; (b) example FPL of size 8 and (c) corresponding ASM; (d) correspondence rules

FPLs are in bijection with several different families of discrete objects, the most prominent in the mathematics literature being alternating-sign matrices of the same size. An alternating-sign matrix has entries 0,1 and -1 , with the condition that, in each line and column, nonzero entries alternate in sign,

(i) Here $N$ refers to the number of vertices on each side; vertices are given matrix-like coordinates $(i, j)$ with $0 \leq i, j \leq N-1$, the top left vertex having coordinates $(0,0)$ 
starting and ending with a 1 . This correspondence is sketched in Figure 1; the "even" and "odd" rules refer to the parity of the sum of line and column indices. Other objects include configurations of the square ice or 6-vertex model [19].

The enumeration formula for alternating-sign matrices of size $n$ was proved in [27, 14]:

$$
A(n)=\prod_{i=0}^{n-1} \frac{(3 i+1) !}{(n+i) !}
$$

together with $A(1)=1$, this is equivalent to the recurrence $A(n+1)=A(n) \cdot n !(3 n+1) ! /((2 n) !(2 n+1))$.

The group of isometries of the square acts naturally on alternating-sign matrices and FPLs (with the caveat that some isometries, depending on the parity of $N$, may exchange the "in" and "out" boundary edges, so that to have a given isometry act on FPLs one may have to take the complement of the set of edges; for rotations, this only happens when one performs a quarter-turn on FPLs of odd size). As a result, for each subgroup of the full group of isometries one may consider a symmetry class of FPLs, which is the set of FPLs that are invariant under the whole subgroup. Enumeration formulae have been conjectured [23] for many classes, and some of them have been proved [15, 22, 21]. In this paper, we are only concerned with two classes: FPLs that are invariant under a half-turn rotation (HTFPLs), and FPLs that are invariant under a quarter-turn rotation (QTFPLs).

While HTFPLs of all sizes exist, QTFPLs are a slightly different matter. QTFPLs of all odd sizes exist, but because for odd sizes the 90 degree rotation exchanges the boundary conditions, QTFPLs are actually self-complementary (as edge sets) rather than invariant under the rotation. QTFPLs of even size $N$ only exist if $N$ is a multiple of 4 , which is easiest seen on the corresponding alternating-sign matrices: the sum of entries in any quarter of the square has to be exactly a quarter of the sum of all entries in the matrix, which is equal to $N$.

For $N=4 n+2$, while there are no QTFPLs of size $N$, we can define the closest thing to it, which we call "quasi-quarter-turn invariant FPLs" (qQTFPLs), and define as follows: an FPL of size $N=4 n+2$ is a qQTFPL if its symmetric difference with its image under a 90 degree rotation is reduced to a single 4-cycle at the center of the grid; furthermore, we require that a qQTFPL contain the two horizontal edges of this center cycle.

The alternating-sign matrices corresponding to qQTFPLs are exactly those which have quarter-turn invariance except for the four center entries $\left(a_{2 n, 2 n}, a_{2 n+1,2 n}, a_{2 n, 2 n+1}, a_{2 n+1,2 n+1}\right)$, which must take respective values $(1,0,0,1)$ or $(0,-1,-1,0)$. This particular class of ASMs does not seem to have been considered previously in the literature, and their enumerating sequence does not appear in the Online Encyclopedia of Integer Sequences [24].

Robbins [23] conjectured, and Kuperberg [15] proved, among other things, that the numbers of FPLs of size $N$, HTFPLs of size $2 N$, and QTFPLs of size $4 N$, are bound by the very intriguing formula

$$
A_{\mathrm{QT}}(4 N)=A_{\mathrm{HT}}(2 N) A(N)^{2} \text {; }
$$

based on exhaustive enumeration up to $N=4$, we conjecture the following similar formula:

Conjecture 1 The number of qQTFPLs of size $4 N+2$ is

$$
A_{\mathrm{QT}}(4 N+2)=A_{\mathrm{HT}}(2 N+1) A(N+1) A(N) .
$$

Actually, a refined identity seems to hold, which nicely extends a further conjecture of Robbins: 
Conjecture 2 Let $A(n ; y)$ (respectively, $A_{\mathrm{HT}}(n ; y), A_{\mathrm{QT}}(n ; y)$ ) denote the enumerating polynomial of FPLs (respectively, HTFPLs, qQTFPLs) of size $n$; each object is given weight $y^{k}$, where $k$ is the index of the column (numbered 0 to $n-1$ ) containing the single nonzero entry in the first line of the corresponding alternating-sign matrix; then for any $n \geq 1$,

$$
A_{\mathrm{QT}}(4 n+2 ; y)=y A_{\mathrm{HT}}(2 n+1 ; y) A(n+1 ; y) A(n ; y) .
$$

\subsection{Link patterns}

Any FPL $f$ of size $N$ has a link pattern, which is a partition of the set of integers 1 to $2 N$ into pairs, defined as follows: first label the endpoints of the open loops 1 to $2 N$ in clockwise or counterclockwise order (for definiteness, we use counterclockwise order, starting with the top left endpoint); then the link pattern will include pair $\{i, j\}$ if and only if the FPL contains a loop whose two endpoints are labeled $i$ and $j$. Because the loops are noncrossing, the link pattern satisfies the noncrossing condition: if a link pattern contains two pairs $\{i, j\}$ and $\{k, \ell\}$, then one cannot have $i<k<j<\ell$. The possible link patterns for FPLs of size $N$ are counted by the Catalan numbers $C_{N}=\frac{1}{N+1}\left(\begin{array}{c}2 N \\ N\end{array}\right)$, and an easy encoding of link patterns by Dyck words (or well-formed parenthese words) is as follows: if $\{i, j\}$ is one of the pairs of the pattern with $i<j$, the $i$-th letter of the Dyck word is an $a$ (which stands for an opening parenthese) while the $j$-th letter is a $b$ (closing parenthese).

If an FPL is invariant under a half-turn rotation, then clearly its link pattern $\pi$ has a symmetry property: if $\{i, j\} \in \pi$, then $\{i+N, j+N\} \in \pi$ (taking integers modulo $2 N$ ). If $N$ is odd, the partition is into an odd number of pairs, and exactly one pair will be of the form $\{i, i+N\}$; if $N$ is even, no pair of the form $\{i, i+N\}$ will be present. This symmetry lets one encode a half-turn-invariant link pattern with a word $w$ of length $N$ instead of $2 N$, as follows: for $1 \leq i \leq N$, if $i$ is matched with $j$ with $i<j<j+N$, then the $i$-th letter is an $a$; if $i$ is matched with $i+N($ odd $N)$, then the $i$-th letter is a $c$; otherwise, $i$ is matched with $j$ where $j<i$ or $j>i+N$, and the $i$-th letter is a $b$.

It is easy to check that, for even $N$, the word $w$ has $N / 2$ occurrences of $a$ and $b$, and is thus a bilateral Dyck word, while for odd $N$, it has exactly one occurrence of $c$ and $(N-1) / 2$ occurrences of each of $a$ and $b$, and is of the form $w=u c v$. In this case, $v u$ has to be a Dyck word. Overall, the total number of possible link patterns is counted by the unified formula $\frac{N !}{N / 2 \mid ! N / 2\rceil !}$.

The $2 N$ generators $e_{1}, \ldots, e_{2 N}$ of the cyclic Temperley-Lieb algebra act on link patterns of size $N$ FPLs in the following way: if link pattern $\pi$ contains pairs $\{i, j\}$ and $\{i+1, k\}$, then $e_{i} \pi=\pi^{\prime}$, where $\pi^{\prime}$ is obtained from $\pi$ by replacing the pairs $\{i, j\}$ and $\{i+1, k\}$ by $\{i, i+1\}$ and $\{j, k\}$; if $\{i, i+1\} \in \pi$, then $\pi^{\prime}=\pi$. One easily checks that the $e_{i}$ operators satisfy the Templerley-Lieb commutation relations

$$
e_{i}^{2}=e_{i}, \quad e_{i} e_{i \pm 1} e_{i}=e_{i \pm 1} e_{i} e_{i \pm 1}, \quad e_{i} e_{j}=e_{j} e_{i} \text { when }|i-j|>1
$$

(generator indices, just like integers in the link pattern, are used modulo $2 N$ ). Similarly, the $N$ "symmetrized" operators $e_{i}^{\prime}=e_{i} e_{i+N}$ (for $N \geq 2$ ) act on the link patterns of HTFPLs of size $N$, and these $N$ symmetrized operators also satisfy the commutation relations for the $N$-generator cyclic Temperley-Lieb algebra.

In both the nonsymmetric and half-turn-symmetric cases, one can define a Markov chain on link patterns where, at each time step, one of the appropriate generators is chosen uniformly at random and applied to the current state. In each case, the Markov chain is easily checked to be irreducible and aperiodic, hence it has a unique stationary distribution. Recent interest in FPLs and their link patterns is largely due to the following conjectures; see $[9,18,28]$ for recent advances, and the bibliography in [25] : 
Conjecture 3 (Razumov, Stroganov [20]) The stationary distribution for link patterns of size $N$ is

$$
\mu(\pi)=\frac{A(N ; \pi)}{A(N)} .
$$

Conjecture 4 (de Gier [7, 17]) The stationary distribution for half-turn-invariant link patterns of size $N$ is

$$
\mu_{\mathrm{HT}}(\pi)=\frac{A_{\mathrm{HT}}(N ; \pi)}{A_{\mathrm{HT}}(N)} .
$$

By their definitions, the stationary distributions $\mu$ and $\mu_{\mathrm{HT}}$ are invariant under the "rotation" mapping (in the noncrossing partition view) $i \mapsto i+1 \bmod 2 N$. Wieland [26] bijectively proved that the distribution of link patterns of FPLs also has this property; his bijection maps HTFPLs to HTFPLs and (even-sized) QTFPLs to QTFPLs, so the same is true of the distributions of their link patterns. It is easy to check that the same bijection maps qQTFPLs to qQTFPLs (with the special provision that it might change the edges around the center square from "two horizontal edges" to "two vertical edges", so the edges around this center square might have to be inverted).

\section{Link patterns of QTFPLs}

Let $N=4 n$. Any QTFPL $f$ of size $N$ is also a HTFPL, so its link pattern can be described by a bilateral Dyck word $w$ of length $N$. But, because $f$ is invariant under a quarter-turn rotation, $w w$ must be invariant under conjugation with its left factor $w^{\prime}$ of length $N / 2$. This means we must have $w=w^{\prime} \cdot w^{\prime}$, and thus $w^{\prime}$ is also a bilateral Dyck word.

Thus, the link patterns of QTFPLs of size $4 n$ can be described by the same words that we use to describe link patterns of HTFPLs of size $2 n$. We use $\mathcal{A}_{\mathrm{QT}}(N ; w)$ (where $N$ is divisible by 4 , and $w$ is a bilateral Dyck word of length $N / 2$ ) to denote the set of all QTFPLs of size $N$ with link pattern $w$ (or link pattern $w . w$ when viewed as HTFPLs), and $A_{\mathrm{QT}}(N ; w)$ to denote its cardinality.

We conjecture the following:

Conjecture 5 For any $n \geq 0$ and bilateral Dyck word $w$ of length $2 n$,

$$
A_{\mathrm{QT}}(4 n ; w)=A_{\mathrm{HT}}(2 n ; w) \cdot A(n)^{2} .
$$

In other words, the link patterns of even-sized QTFPLs are distributed exactly as those of HTFPLs with half their size.

Conjecture 5 has been checked by exhaustive enumeration up to $k=5$ (there are 114640611228 QTFPLs of size 20; the next term in the sequence is 10995014015567296 , which makes exhaustive generation unreasonable).

When $f \in \mathcal{A}_{\mathrm{QT}}(4 n+2)$ is a qQTFPL, it is also a HTFPL and its link pattern as such is described by a bilateral Dyck word of length $4 N+2$. But, again, the link pattern is of a special form: because of the rotational symmetry, the paths entering the center square of the grid by its four corners are rotational images of each other, and cannot form closed loops. Thus, these paths exit the grid at 4 endpoints, which form a single orbit under the quarter-turn rotation. Furthermore, the HTFPL link pattern is necessarily of the form uavubv or ubvuav, where $v u$ is a Dyck word of length $2 n$ (this implies that the factorization is unique). If we retain only the first $2 n+1$ letters of this word, and replace the distinguished $a$ or $b$ letter 
with a $c$, what we obtain is exactly the link pattern of a HTFPL of sized $2 n+1$; this is what we hereafter call the link pattern of $f$. Note that if, in the definition of qQTFPLs, we required that the center square have vertical edges instead of horizontal edges, this would only change link patterns as HTFPLs (patterns of the form $u a v u b v$ would become $u b v u a v$ would become $u a v u b v$, and vice versa) but not as qQTFPLs.

As an example, the qQTFPL shown in Figure 2(a) has link pattern babca as a qQTFPL, babaababba as a HTFPL, and $a a b a a b a b b a b a b a a b a b b b$ as a full FPL link pattern.

With this convention, we have a conjecture for the link patterns of qQTFPLs of size $4 n+2$, relating them to those of HTFPLs of size $2 n+1$ :

Conjecture 6 For any $n \geq 0$ and any half-turn-invariant link pattern $w$ of length $2 n+1$,

$$
A_{\mathrm{QT}}(4 n+2 ; w)=A_{\mathrm{HT}}(2 n+1 ; w) A(n+1) A(n) .
$$

Of course, summation over all link patterns gives Conjecture 1, and this can be interpreted as saying that link patterns of qQTFPLs of size $4 n+2$ are distributed exactly as those of HTFPLs of size $2 n+1$.

Conjectures 1, 2 and 6 have been checked by exhaustive enumeration up to $n=4$; the total number of qQTFPLs of size 18 is $39204 \cdot 429 \cdot 42=706377672$. The next term in the conjectured sequence, $7422987 \cdot 7436 \cdot 429=23679655141428$, is out of reach of exhaustive enumeration programs.

A note on terminology: in the rest of this paper, whenever we mention the link pattern of a QTFPL or qQTFPL, it should be understood to mean the word with length half the size of the FPL; if we need to reference the link pattern as an FPL (which is a Dyck word with length double the size of the FPL), we will write full link pattern.

\section{A special case: the rarest link pattern}

In this section, we prove special cases of Conjectures 5 and 6 when the considered link pattern is a very specific one. When the wanted link pattern is of the form $b^{n} a^{n}$ (for QTFPLs) or $b^{n} c a^{n}$ (for qQTFPLs), the HTFPLs whose enumeration appear in the conjectures have full link pattern $a^{2 n} b^{2 n}$ or $a^{2 n+1} b^{2 n+1}$, respectively. In each case, there is only one HTFPL with such a full link pattern; in fact, there is only one FPL with such a link pattern (this has been noticed by many authors; one easy way to properly prove it is with the fixed edge technique of de Gier which we use below). Thus, to prove the corresponding special cases of Conjectures 5 and 6, we only need to prove that the corresponding QTFPLs and qQTFPLs are counted by $A(n)^{2}$ and $A(n) A(n+1)$, respectively. Both proofs are through a bijection with a specific class of plane partitions.

For our purposes, a plane partition of size $k$ is a tiling of the regular hexagon $H_{k}$ of side $k$ with rhombi of unit side. When the hexagon is tiled with equilateral triangles of unit side, the dual graph is a region $R_{k}$ of the honeycomb lattice, and rhombus tilings are in natural bijection with perfect matchings of $R_{k}$.

A plane partition is said to be cyclically symmetric if the tiling is invariant under a rotation of 120 degrees, and self-complementary if it is invariant under a central symmetry (the terminology is somewhat confusing when plane partitions are viewed as tilings, but it is standard). Thus, cyclically symmetric, self-complementary plane partitions (CSSCPPs for short) are those that are invariant under a rotation of 60 degrees. It is easy to see that CSSCPPs only exist for even sizes, and it is known that the number of CSSCPP of size $2 n$ is equal to $A(n)^{2}$.

We define a quasi-cyclically symmetric, self-complementary plane partition (qCSSCPP) of size $2 n+1$ as a rhombus tiling, invariant under rotation of 60 degrees, of the regular hexagon of size $2 n+1$ with 


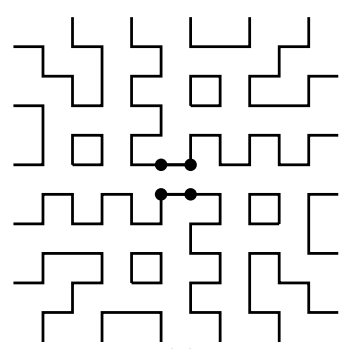

(a)

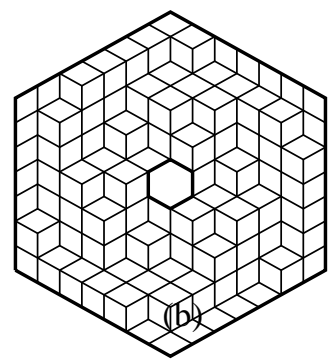

Fig. 2: (a) Example qQTFPL of size 10 and (b) Example qCSSCPP of size 7

the central unit side hexagon removed. Such tilings (with a hole of a possibly different size) appear as punctured plane partitions in [8], where the connection with cyclically symmetrics tilings of a hexagon with a triangular hole [6] is noticed.

We will prove the following:

Theorem 7 For any $n \geq 1$, there is a bijection between $\mathcal{A}_{\mathrm{QT}}\left(4 n ; b^{n} a^{n}\right)$ and the set of CSSCPPs of size $2 n$, and a bijection between $\mathcal{A}_{\mathrm{QT}}\left(4 n+2 ; b^{n} c a^{n}\right)$ and the set of $q C S S C P P s$ of size $2 n+1$.

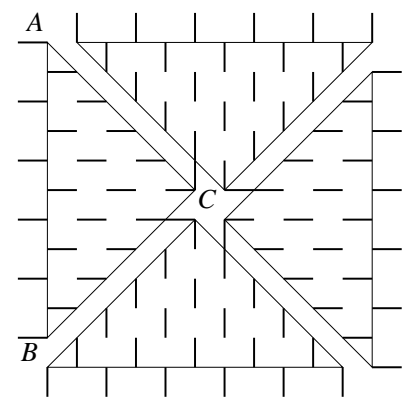

(a)

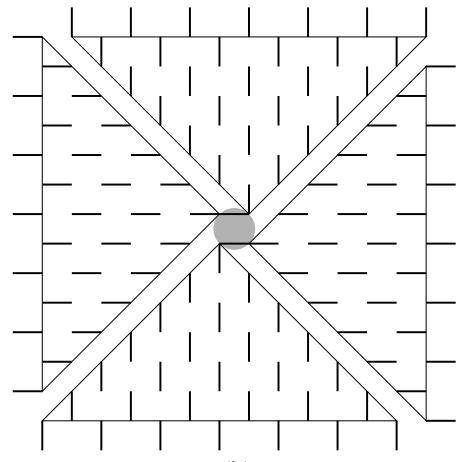

(b)

Fig. 3: Fixed edges for (a) $\mathcal{A}_{\mathrm{QT}}(12$, bbbaaa $)$ (b) $\mathcal{A}_{\mathrm{QT}}(14$, bbbcaaa $)$

The known enumeration of CSSCPPs then concludes the proof of (5) for pattern $b^{n} a^{n}$; to prove (6) for pattern $b^{n} c a^{n}$, we will need our last theorem:

Theorem 8 The number of $q$ CSSCPPs of size $2 n+1$ is $A(n) A(n+1)$.

Proof of Theorem 7: We rely on the technique of "fixed edges" due to de Gier [7], as used by Caselli and Krattenthaler in [2] (see also [10,3]). The technique uses the fact that, for a given link pattern, there may be a large set of edges which appear in all FPLs with this particular link pattern. In some cases, this makes 
it possible to find a bijection between the target set of FPLs and the perfect matchings of some particular planar graph, typically a region of the hexagonal lattice.

QTFPLs with link pattern $b^{n} a^{n}$ have the full link pattern $a^{n}\left(a^{n} b^{n}\right)^{3} b^{n}$, which consists of 4 sets of $n$ nested arches each. This means that, on each of the grid sides, all $n$ outgoing links are forbidden from connecting to each other; thus, by Lemma 3.1 of [3], the following edges are fixed in every FPL $f \in \mathcal{A}\left(4 n ; a^{n}\left(a^{n} b^{n}\right)^{3} b^{n}\right):$

- each horizontal edge whose left endpoint has odd sum of coordinates, inside the triangle whose vertices have coordinates $(0,0),(4 n-2,0)$ and $(2 n-1,2 n-1)$ (triangle $A B C$ on Figure 3(a));

- their orbits under the action of the 90 degree rotation centered at $(2 n-1 / 2,2 n-1 / 2)$.

Similarly, qQTFPLs with link pattern $b^{n} c a^{n}$ have as their full link pattern $a^{2 n+1} b^{n} a^{n+1} b^{n+1} a^{n} b^{2 n+1}$ (four sets of nested arches, with alternatingly $n+1, n, n+1$ and $n$ arches each). Again, the same fixed edges appear, as shown in Figure 3(b).

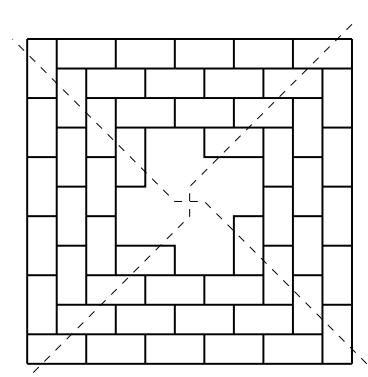

(a)

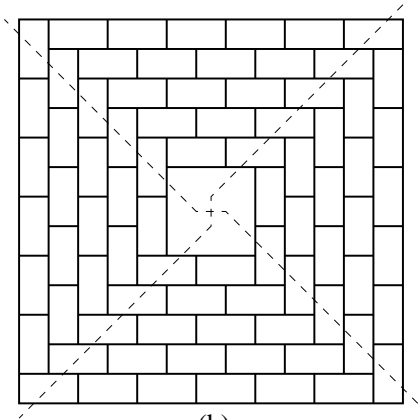

(b)

Fig. 4: Graphs of non-fixed edges for (a) $\mathcal{A}_{\mathrm{QT}}\left(12\right.$, bbbaaa) (b) $\mathcal{A}_{\mathrm{QT}}(14$, bbbcaaa $)$

In a QTFPL of size $4 n$, the four "center" edges joining vertices $C$ and its rotational images must either all be included, or all excluded; with these vertices already having one incident fixed edge each, they must be excluded. This in turn forces the presence of four more fixed edges, as shown in Figure 3(a). (The symmetry conditions also force additional edges in the corners, but it is slightly more convenient to not mention them now.)

To prove that we indeed have a bijection, we need to check that all QTFPLs (respectively, qQTFPLs) sharing the above-mentioned edges have link pattern $b^{n} a^{n}$ ) (respectively, $b^{n} c a^{n}$ ). We do this in detail for the QTFPL case; the proof for qQTFPL is similar.

Consider the $2 n$ paths starting from endpoints along segment $A B$. The horizontal fixed edges inside triangle $A B C$ prevent them from connecting with each other, so that each of them will exit triangle $A B C$ either to the top (through segment $A C$, including $C$ but excluding $A$ ) or to the bottom (through segment $C B$, including $B$ but excluding $C$. Any path exiting through segment $A C$ will be connected to one from the top border, while any path exiting through segment $C B$ will be connected to one from the bottom border. Thus, the link pattern will be of the form $b^{k} a^{2 n-k}$, where $k$ is the number of paths exiting along segment $A C$. But quarter-turn symmetry implies that the number of paths exiting triangle $A B C$ 
through segment $A C$ is equal to the number of paths entering triangle $A B C$ from the bottom triangle; thus, $k=2 n-k$, and the link pattern is indeed $b^{n} a^{n}$.

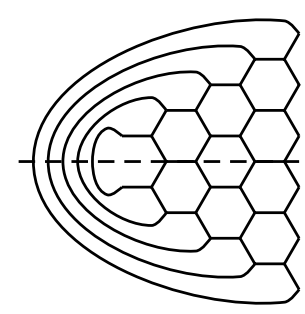

(a) $G_{6}$

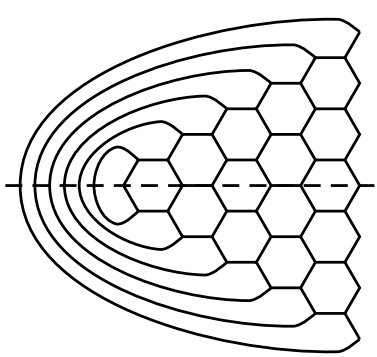

(b) $G_{7}$

Fig. 5: Quotients under rotation of the nonfixed edge graphs in Figure 4

Note that in both the QTFPL and qQTFPL cases, each vertex in the grid is incident to either 1 or 2 fixed edges. Thus, if we delete from the grid the fixed edges and the "forbidden" edges (those non-fixed edges that are incident to at least one vertex with two incident fixed edges), we get a graph whose rotationally invariant perfect matchings are in bijection with the considered symmetric FPLs. These two graphs, shown on Figure 4, naturally have a rotational symmetry of order 4, so we need only consider the perfect matchings of their orbit graphs under this rotational symmetry, which are shown on Figure 5 (the dashed lines in Figure 4 show where to "cut" to obtain the quotients). For QTFPLs, this quotient graph is exactly the orbit graph, under rotational symmetry of order 6 , of the honeycomb graph $R_{2 n}$; for qQTFPLs, it is the orbit graph, under rotational symmetry of order 6 , of the "holed" honeycomb graph $R_{2 n+1}^{\prime}$. Putting all pieces together, we have the required bijections between FPLs and plane partitions.

Proof of Theorem 8: We now turn to the enumeration of qCSSCPPs of size $2 n+1$, for which we know that they are in bijection with the perfect matchings of the quotiented honeycomb lattice region $G_{2 n+1}$.

Notice that $G_{2 n+1}$, as shown in Figure 5, has a reflective symmetry, with $2 n$ vertices on the symmetry axis. Thus, we can use Ciucu's Matching Factorization Theorem [4] (or, rather, the slight generalization proved in Section 7 of [4], and used, in a very similar context to ours, in [5]), and we get that the number of perfect matchings of $G_{2 n+1}$ is $2^{n} M^{*}\left(G_{2 n+1}^{\prime}\right)$, where $G_{2 n+1}^{\prime}$ is $G_{2 n+1}$ with all edges incident to the symmetry axis, and lying below it, removed, and edges lying on the symmetry axis weighted $1 / 2$; and $M^{*}(G)$ denotes the weighted enumeration of perfect matchings of $G$, that is, the sum over perfect matchings of the product of weights of selected edges.

$G_{2 n+1}^{\prime}$, when redrawn as a region of the honeycomb lattice, is the dual of region $R_{2 n+1}^{\prime}$ of the triangular lattice (shown on Figure 6(b), with the weight $1 / 2$ rhombi greyed), on which we need to count rhombi tilings. $n+1$ rhombi on the right border of $R_{2 n+1}^{\prime}$ are fixed (will appear in all tilings). Using a classical correspondence between rhombi tilings and weighted configurations of nonintersecting lattice paths, we are left with counting the number of nonintersecting (square) lattice path configurations, where the paths, using East and South unit steps, collectively join vertices $A_{i}, 0 \leq i \leq n-1$, to vertices $B_{j}, 0 \leq j \leq n-1$, with respective coordinates $(i, 2 i+2)$ and $(2 j, j)$; the horizontal edges with right endpoints $B_{j}$ carry a 


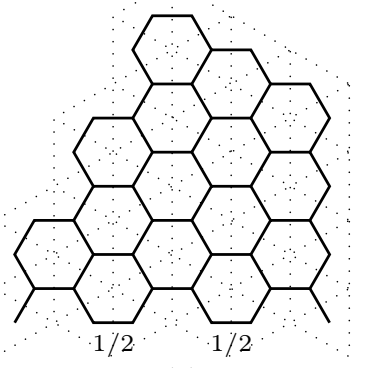

(a)

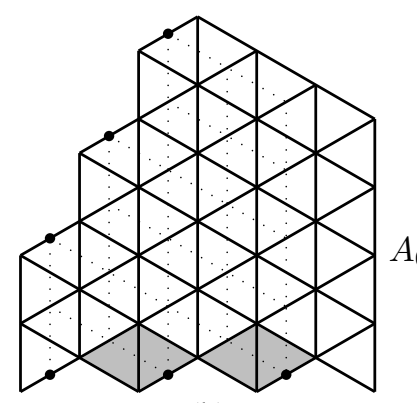

(b)
$A_{2}$

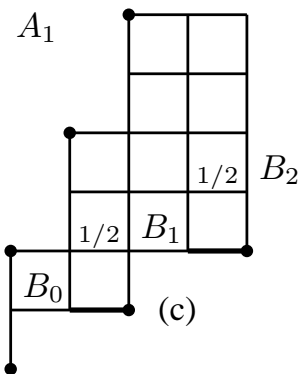

Fig. 6: (a) Honeycomb region $G_{7}^{\prime}$, (b) Triangular lattice $R_{7}^{\prime}$ and (c) corresponding square lattice points

weight $1 / 2$, so that the weighted enumeration of paths joining vertices $A_{i}$ and $B_{j}$ is

$$
w\left(A_{i}, B_{j}\right)=\frac{1}{2}\left(\begin{array}{c}
i+j+1 \\
2 j-i
\end{array}\right)+\left(\begin{array}{c}
i+j+1 \\
2 j-i-1
\end{array}\right)=\frac{1}{2}(3 i+4) \frac{(i+j+1) !}{(2 j-i) !(2 i-j+2) !} .
$$

The Lindström-Gessel-Viennot theorem $[16,12]$ now expresses $M^{*}\left(G_{2 n+1}^{\prime}\right)$ as the determinant

$$
M^{*}\left(G_{2 n+1}^{\prime}\right)=\operatorname{det}\left(w\left(A_{i}, B_{j}\right)\right)_{0 \leq i, j \leq n-1} ;
$$

factoring out $\frac{3 i+4}{2}$ in line $i$ of the matrix, we get the number of perfect matchings of $G_{2 n+1}$ as

$$
M\left(G_{2 n+1}\right)=\left(\prod_{i=0}^{n-1} 3 i+4\right) \operatorname{det}\left(\frac{(i+j+1) !}{(2 j-i) !(2 i-j+2) !}\right)_{0 \leq i, j \leq n-1} .
$$

The determinant in (8) happens to be the special case $x=2, y=0$ of [13, Theorem 40] (the enumeration of CSSCPPs of size $2 n$ by the same method, as in [5, 6], corresponds to $x=1, y=0$ ). Factoring in the product, this yields

$$
M\left(G_{2 n+1}\right)=\prod_{i=0}^{n-1} \frac{i !(i+1) !(3 i+1) !(3 i+4) !}{(2 i) !(2 i+1) !(2 i+2) !(2 i+3) !} .
$$

Using this expression, one easily checks that $M\left(G_{2 n+1}\right) / M\left(G_{2 n-1}\right)=A(n+1) / A(n-1)$, thus proving the theorem by induction (the case $n=1$ corresponds to checking that there are only 2 qCSSPPs of size 3 ).

\section{Further comments}

The starting point and first motivation for this paper, as the title suggests, was Conjecture 5 , which nicely complements the previous conjectures of Razumov-Stroganov and de Gier. This suggests that a combinatorial proof of one of the conjectures might be adapted to yield proofs of all of them, possibly by explicitly 
devising operators on FPLs that project to the $e_{i}$ operators on link patterns, while having suitable bijective properties.

The definitions of qQTFPLs and qCSSCPPs evolved out of an attempt to devise a general framework for the random generation of symmetric FPLs and plane partitions [11]; their enumerative properties came as a total surprise.

A proof of Conjecture 1, using techniques inspired from [15, 21], will be given in an upcoming paper [1].

\section{Acknowledgements}

The author is grateful to Philippe Nadeau for fruitful discussions and bibliographic pointers in early stages of this work, and to Paul Zinn-Justin and Christian Krattenthaler for helpful comments on a previous version of this paper.

\section{References}

[1] J.-C. Aval and P. Duchon. Enumeration of (quasi-)quarter-turn symmetric alternating sign matrices of even size. in preparation.

[2] F. Caselli and C. Krattenthaler. Proof of two conjectures of Zuber on fully packed loop configurations. J. Combin. Theory Ser. A, 108:123-146, 2004.

[3] F. Caselli, C. Krattenthaler, B. Lass, and P. Nadeau. On the number of fully packed loop configurations with a fixed associated matching. Elec. J. Combin., 11(2):R16, 2005.

[4] M. Ciucu. Enumeration of perfect matchings in graphs with reflective symmetry. J. Combin. Theory Ser. A, 77:67-97, 1997.

[5] M. Ciucu. The equivalence between enumerating cyclically symmetric, self-complementary and totally symmetric, self-complementary plane partitions. J. Combin. Theory Ser. A, 86:382-389, 1999.

[6] M. Ciucu and C. Krattenthaler. Plane partitions II: $51 / 2$ symmetry classes. Advanced Studies in Pure Mathematics, 28:83-103, 2000.

[7] J. de Gier. Loops, matchings and alternating-sign matrices. Discr. Math., 298:365-388, 2005.

[8] J. de Gier, P. Pyatov, and P. Zinn-Justin. Punctured plane partitions and the $q$-deformed KnizhnikZamolodchikov and Hirota equations. Available as arXiv:math . CO/0712 . 3584.

[9] P. Di Francesco and P. Zinn-Justin. Around the Razumov-Stroganov conjecture: proof of a multiparameter sum rule. Electronic J. Combin., 12:R6, 2005.

[10] P. Di Francesco, P. Zinn-Justin, and J.-B. Zuber. A bijection between classes of fully packed loops and plane partitions. Electronic J. Combin., 11(1):R64, 2004.

[11] P. Duchon and F. Le Gac. Random generation of symmetric and quasi-symmetric alternating-sign matrices. To appear in GASCOM 2008. 
[12] I. Gessel and X. Viennot. Binomial determinants, paths and hook formulae. Adv. Math., 58:300-321, 1985.

[13] C. Krattenthaler. Advanced determinant calculus. Séminaire Lotharingien de Combinatoire, 42:B42q, 1999

[14] G. Kuperberg. Another proof of the alternating sign matrix conjecture. Internat. Math. Res. Notices, 1996:139-150, 1996.

[15] G. Kuperberg. Symmetry classes of alternating-sign matrices under one roof. Annals of Mathematics, 156:835-866, 2002.

[16] B. Lindström. On the vector representations of induced matroids. Bull. London Math. Soc., 5:85-90, 1973.

[17] S. Mitra, B. Nienhuis, J. de Gier, and M.T. Batchelor. Exact expressions for correlations in the ground state of the dense $O(1)$ loop model. J. Stat. Mech: Theor. Exper., P09010:24pp., September 2004.

[18] V. Pasquier. Quantum incompressibility and Razumov Stroganov type conjectures. Ann. Inst. Henri Poincaré, 7:397-421, 2006.

[19] J. Propp. The many faces of alternating-sign matrices. In Discrete Models: Combinatorics, Computation, and Geometry, volume AA of DMTCS Proceedings, pages 43-58, 2001.

[20] A.V. Razumov and Yu.G. Stroganov. Combinatorial nature of ground state of $O(1)$ loop model. Theor. Math. Phys., 138:333-337, 2004.

[21] A.V. Razumov and Yu.G. Stroganov. Enumeration of quarter-turn-symmetric alternating sign matrices of odd order. Theoretical and Mathematical Physics, 149(3):1639-1650, 2006.

[22] A.V. Razumov and Yu.G. Stroganov. Enumerations of half-turn-symmetric alternating-sign matrices of odd order. Theoretical and Mathematical Physics, 148(3):1174-1198, 2006.

[23] D. Robbins. Symmetry classes of alternating sign matrices. Unpublished manuscript arXiv:math.CO/000804.

[24] N.J.A. Sloane. The on-line encyclopedia of integer sequences. Available online at http: //www.research.att.com/ ^njas/sequences/.

[25] J. Thapper. Refined counting of fully packed loop configurations. Séminaire Lotharingien de Combinatoire, 56:B56e, 27pp., 2007.

[26] B. Wieland. A large dihedral symmetry of the set of alternating-sign matrices. Electronic Journal of Combinatorics, 7(1):R37, 2000.

[27] D. Zeilberger. Proof of the alternating sign matrix conjecture. Electronic Journal of Combinatorics, 3(2):R13, 1996.

[28] P. Zinn-Justin. Proof of the Razumov-Stroganov conjecture for some infinite familiers of link patterns. Electronic J. Combin., 13:R110, 2007. 